\title{
Numerical Simulation of Micro-Flow Surface Smoothing of Fused Silica by $\mathrm{CO}_{2}$ Laser
}

\author{
Xinyu Luo ${ }^{1,2}$, Wei Yang ${ }^{1}$, Ting Tan², Dexing Zhu², Jin Zhuo², Mincai Liu³, Qinghua Zhang ${ }^{3}$, and Yaguo Li ${ }^{2, *}$ \\ ${ }^{1}$ School of Aerospace Engineering, Xiamen University, Xiamen 361005, China \\ ${ }^{2}$ Fine Optical Engineering Research Center, Chengdu 610041, China \\ ${ }^{3}$ Laser Fusion Research Center, China Academy of Engineering, Mianyang 621900, China \\ ${ }^{*}$ Corresponding author's e-mail: yargolee@163.com
}

\begin{abstract}
Micro-flow smoothing of fused silica surface with $\mathrm{CO}_{2}$ lasers were numerically simulated. Two types of laser intensity distribution, namely Gaussian and top-hat, were modeled on various surface morphology. In the models, the surface tension and Marangoni effect were taken into account. The results show that surface tension and Marangoni effect acts synergically whereas surface tension dominates the resultant surface in our simulated conditions. Top-hat intensity is conducive to smoothing of fused silica glass. In addition, surfaces with both periodic and random topography can be smoothed. Under the $585.69 \mathrm{~W} / \mathrm{cm}^{2}$ power density, the best roughness (RMS) of Gaussian and top-hat is $2.85 \mathrm{~nm}$ and $2.48 \mathrm{~nm}$ respectively (initial roughness $>700 \mathrm{~nm}$ ).
\end{abstract}

DOI: $10.2961 /$ jlmn.2021.02.2008

Keywords: laser smoothing, fused silica, simulation, surface morphology, roughness

\section{Introduction}

Laser smoothing of fused silica is being paid increasingly attention in recent years due to the non-contact and high speed features of laser smoothing technique [1]. The smoothing laser is, in most cases, $\mathrm{CO}_{2}$ laser of $10.6 \mu \mathrm{m}$ wavelength because fused silica strongly absorbs $10.6 \mu \mathrm{m}$ laser light within the topmost layers of tens of microns. The absorbed laser is mostly converted into heat, resulting in sharp increase in local temperature. The temperature usually can reach as high as softening point or even evaporation temperature. As a consequence, fused silica can be smoothed by means of micro-flow or ablated by evaporation. It is mass loss that differentiates smoothing from ablation. In smoothing regime, mass loss is hardly perceptible while significant material is removed in ablation. In some cases such as drilling, scribing of fused silica glass in microfluidics or photonics, ablation is preferred. On the other hand, smoothing appears attractive in surface figuring without degrading the original surface which is the case of our study. Therefore, the temperature must lie in between softening and evaporation temperature in order to smooth fused silica in micro-flow regime. In micro-flow regime, surface tension and Marangoni take effects synergically, which results in the final surface tomography.

In 1980s, it has already been proved that $\mathrm{CO}_{2}$ laser can recover and smooth scratched glass surface [2,3]. From 2003, a team in Germany has engaged in $\mathrm{CO}_{2}$ laser polishing [4-9]; it is reported that $\mathrm{CO}_{2}$ laser polishing can reduce the initial surface roughness of fused silica samples from $100 \mathrm{~nm}$ to less than $5 \mathrm{~nm}$. The reason is that the thin layer of material on the surface of fused silica is melted by incident laser, resulting in displacement of glass material and thus surface smoothing. Owing to surface tension, the material flows from the peak to valley and thus the surface is smoothed. $\mathrm{CO}_{2}$ laser polishing process is applied to polish spherical and aspheric glass, even free-form surfaces. Compared with the surface polished with traditional meth- ods, the surface polished by laser contains few microdefects. However, the waviness value of laser polishing is relatively high, for spatial wavelength $\lambda>100 \mu \mathrm{m}$. Yuan [10] used $\mathrm{CO}_{2}$ laser to process the surface of fused silica glass (initial surface roughness RMS $>165 \mathrm{~nm}$ ) and obtained a smooth mirror with $\mathrm{RMS}<1 \mathrm{~nm}$. It is found that there are two ways in the fused silica irradiated by $\mathrm{CO}_{2}$ laser, namely micro-flow and ablation. In micro-flow mode, the surface of fused silica is smooth without mass loss, while the material on fused silica surface evaporates in ablation mode. Wang [1] studied the smooth effect of Gaussian and top-hat $\mathrm{CO}_{2}$ laser on the surface of fused sili$\mathrm{ca}$, and found that the surface with initial surface roughness of $500 \mathrm{~nm}$ (RMS) can be smoothed to < $0.5 \mathrm{~nm}$ (RMS) by both light intensity distributions. Some researchers have established a numerical model of heat transfer and flow coupling in metal laser polishing to describe the flow behavior of molten pool, which has revealed the internal mechanism of metal laser polishing [11-15]. He el al. [16] established a transient numerical model based on the finite element method for $\mathrm{CO}_{2}$ polishing fused silica, revealed the basic physical mechanism in the polishing process, and simulated the surface smoothing process of laser polishing. These models take into account the Gaussian distribution of $\mathrm{CO}_{2}$ laser instead of other laser intensity distribution. In addition, the effect of initial surface morphology on final surface quality was seldom investigated in these models.

In this paper, fused silica with various surface morphologies under the irradiation of Gaussian and top-hat laser were studied. The laser fluence (Gaussian and tophat) was set to $585.69 \mathrm{~W} / \mathrm{cm}^{2}$. The final surface roughness was calculated from the models. The simulation results show that top-hat laser can greatly shorten the smoothing time compared with Gaussian laser. The initial smoothing time is $\mathbf{5 0 ~ m s ~ f o r ~ t o p - h a t ~ l a s e r , ~} 70$ ms for Gaussian laser. As to various surface morphologies, all of them can be smoothed according to our results. The smoothing effect of 
periodic topography under the condition of period $\mathrm{T}=5 \mu \mathrm{m}$ and amplitude $A=1 \mu \mathrm{m}$ is better than others, the optimal roughness (RMS) smoothed by Gaussian and top-hat laser is $2.85 \mathrm{~nm}$ and $2.48 \mathrm{~nm}$ respectively. Unfortunately, although the random topography can be also smoothed, the final effect is not satisfactory, the surface roughness (RMS) reduced from $700 \mathrm{~nm}$ to $520 \mathrm{~nm}$ under the irradiation of Gaussian and top-hat laser.

\section{Simulation model}

Laser smoothing is a multiphysics process, including heat transfer with phase change and fluid flow [16]. A multi-physics model describing the heat transfer and fluid flow process on the fused silica surface was established using a finite element simulation software. The surface temperature of material rises to liquidus temperature and thus forms a molten pool, while fused silica irradiated with laser. Material in molten pool tends to redistribute under the effect of micro-flow to smooth the initial surface roughness that the peak-valley value (PV value) reduced. Also, the final surface morphology tends to be smooth during the process. According to the above mention, the simulation was based on the following assumptions:

1) Fused silica is considered to be isotropic and it is incompressible in liquid state.

2) The simulation temperature is between softening and evaporation temperature.

3) Surface tension and Marangoni effect is considered in the micro-flow mode.

4) Ablation model is neglected due to inconducive to smoothing.

\subsection{Governing equations}

In polishing process, the surface temperature of fused silica will increase after laser irradiation and thus forms a molten pool. The transient temperature is controlled by the governing equations [1] of micro-flow simulation model. The governing equations include heat conduction equation describing temperature field and momentum equation describing fluid, expressed by Eq. (1).

$$
\rho C_{p}\left[\frac{\partial T}{\partial t}+\nabla \cdot(\vec{u} T)\right]-\nabla \cdot(k \nabla T)=0
$$

Where $\rho$ is the density, $C_{p}$ is the heat capacity, $k$ is the thermal conductivity, $\vec{u}$ is the velocity field in the molten pool which can be calculated by Navier-Stokes equation, as shown in Eq.(2):

$$
\rho \frac{\partial \vec{u}}{\partial t}+\rho(\vec{u} \cdot \nabla) \vec{u}=\nabla \cdot\left[-P \vec{I}+\mu\left(\nabla \vec{u}+(\nabla \vec{u})^{T}\right)\right]+F
$$

Where $P$ is the pressure in the molten pool, $\vec{I}$ is the unit matrix, $\mu$ is the dynamic viscosity and $F$ is the volume force, including buoyancy force and gravity. In addition, fused silica is incompressible in liquid state:

$$
\nabla \cdot \vec{u}=0
$$

The small region on the fused silica surface under laser irradiation transforms from solid to liquid state and thus forms a molten pool. Phase transition can happen in this process, which will influence the heat capacity of fused silica. Here, the heat capacity is set as a function of the temperature [17]. Similarly, the heat change will certainly affect the viscosity which is temperature dependent. The viscosity was approximated by Eq. (5), referring to the experimental data reported by Protasov [18].

$$
\begin{gathered}
C_{p}(T)= \begin{cases}-120+4.56 T-\left(7.38 \times 10^{-3}\right) T^{2} \\
+\left(6.59 \times 10^{-6}\right) T^{3}-\left(3.05 \times 10^{9}\right) T^{4} & 273 K<T<1500 K \\
678-0.142 T+\left(3.45 \times 10^{-4}\right) T^{2} & 1500 K<T<1696 K \\
1429 & 1696 K<T\end{cases} \\
\eta= \begin{cases}3.8 \times 10^{-14}(P a \cdot s) \exp \left(\frac{85640 K}{T}\right) & T<1670 K \\
5.8 \times 10^{-14}(P a \cdot s) \exp \left(\frac{61990 K}{T}\right) & T \geq 1670 K\end{cases}
\end{gathered}
$$

\subsection{Boundary conditions}

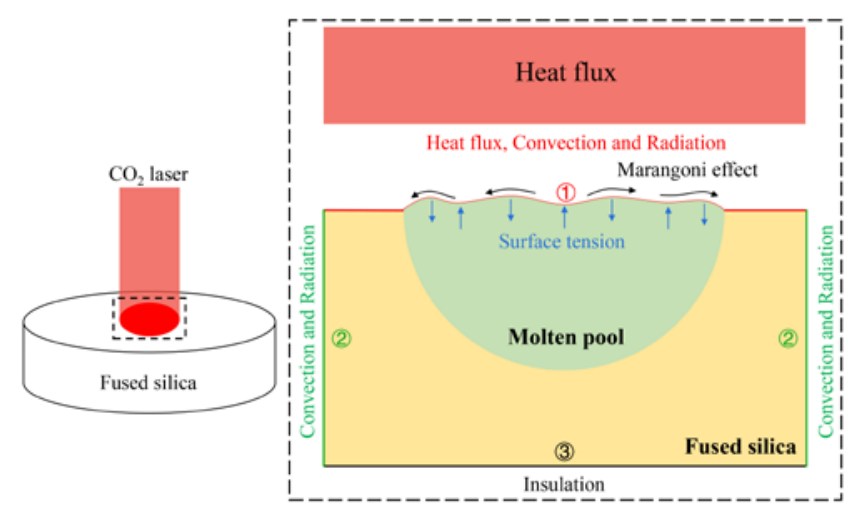

Fig.1 Schematic diagram of simulation model.

As shown in Fig.1, multiple boundary conditions exist in the simulation model describing the $\mathrm{CO}_{2}$ laser polishing process. Boundary condition (1), which includes laser heat flux, convection and surface-to-ambient radiation, can be described in Eq.(6):

$$
-k \nabla T=Q+h\left(T-T_{a}\right)+\varepsilon B\left(T^{4}-T_{a}^{4}\right)
$$

where h is the natural convection coefficient, $\varepsilon$ is the emissivity, $B$ is the Stefan-Boltzmann constant, and Ta is the ambient temperature. Laser heat flux $\mathrm{Q}$ of Gaussian and top-hat distribution is given by Eq. (7) and Eq. (8), respectively:

$$
\begin{aligned}
Q_{\text {Gaussian }} & =\frac{2 P_{\text {Gaussian }}}{\pi r^{2}} \exp \left(\frac{-2 x^{2}}{r^{2}}\right) \\
Q_{\text {Top-hat }} & =\frac{P_{\text {Top-hat }}}{\pi r^{2}}
\end{aligned}
$$

where $\mathrm{P}$ is the laser power, $\mathrm{r}$ is the radius of laser beam, $\mathrm{x}$ is the distance from the center of the laser beam.

The heat exchange between fused silica and its surroundings can be expressed as natural convection. Moreover, all objects above absolute zero have thermal radiation. Boundary (2) is affected by convection and surface-toambient radiation as shown in Eq. (9), while boundary (3) is considered thermal insulation in Eq. (11). The convection is expressed by Eq. (10).

$$
-k \nabla T=h\left(T-T_{a}\right)+\varepsilon B\left(T^{4}-T_{a}^{4}\right)
$$




$$
\begin{aligned}
& -k \nabla T=h\left(T-T_{a}\right) \\
& \nabla T=0
\end{aligned}
$$

When the temperature of molten pool reaches the liquidus temperature, the capillary force acting along the normal direction (surface tension) and the thermal capillary flow acting along the surface (Marangoni effect) will affect the surface topography of the molten pool [16].

In the normal direction, the surface tension can be described as

$$
\sigma_{n}=\kappa \gamma \cdot \vec{n}
$$

In the tangential direction, the Marangoni effect can be described as

$$
\sigma_{t}=\frac{\partial \gamma}{\partial T} \nabla_{s} T \cdot \vec{t}
$$

where $\kappa$ is the curvature of the surface profile, $\gamma$ is the surface tension, and $\nabla_{s} T$ is the temperature gradient along the tangential direction of the surface. $\vec{n}$ and $\vec{t}$ are the unit normal and tangential vectors to the local surface. All boundaries in the computational domain are labeled as shown in Fig.1.

\subsection{Parameter setting}

As shown in Fig.2 (a), the central region under laser irradiation can be regarded as the expected molten pool and the other region is regarded as solid substrate. The molten pool is affected by laser parameters (as laser power and laser beam size). It is assumed that phase transformation only occurs in the expected molten pool, which makes it possible to couple heat transfer with fluid flow. At the top of the geometry of fused silica model, a manually drawn curve with periodic and random topography with peaks and valleys represents the initial surface morphology, where the of 'period' and 'amplitude' is shown (Fig.2 (b)).

In previous studies [10], it was found that there are two ways in the fused silica irradiated by $\mathrm{CO}_{2}$ laser, namely micro-flow and ablation. It is weight loss that differentiates smoothing from ablation. In smoothing regime, mass loss is hardly perceptible while significant material is removed in ablation. By adjusting the laser power, the temperature can be controlled at a level between softening and evaporation temperature, smoothing fused silica in micro-flow regime. As reported in our previous work [10], the smooth power density threshold is $339.531 \mathrm{~W} / \mathrm{cm}^{2}$, about $594.178 \mathrm{~W} / \mathrm{cm}^{2}$ for ablation power density threshold. In this study, only the smooth process in micro-flow regime is considered, that is, the simulated laser power density is set between 339.531$594.178 \mathrm{~W} / \mathrm{cm}^{2}$. Both spot radius of Gaussian laser and top-hat laser were set to be $75 \mu \mathrm{m}$. The dimension of molten pool is generally considered to be slightly smaller than laser beam [14], which is $100 \mu \mathrm{m}$ in the simulation model. The power density was set to be $585.69 \mathrm{~W} / \mathrm{cm}^{2}$, which was slightly less than the ablation power density. The geometric model of the molten pool is shown in the Fig.2. The intensity distribution of Gaussian and top-hat laser is shown in the Fig.3. The parameters of the numerical model are given in Table 1.

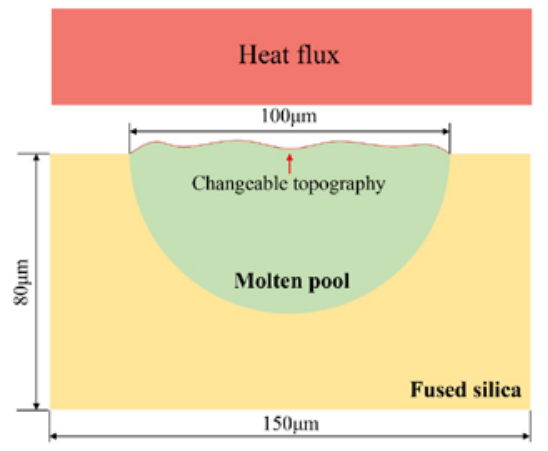

(a)

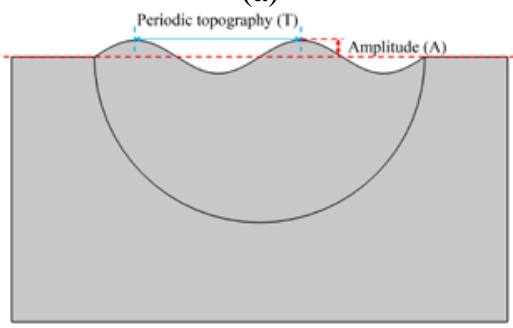

(b)

Fig.2 (a) Geometric model of molten pool (b) Schematic definition of 'period' and 'amplitude'.

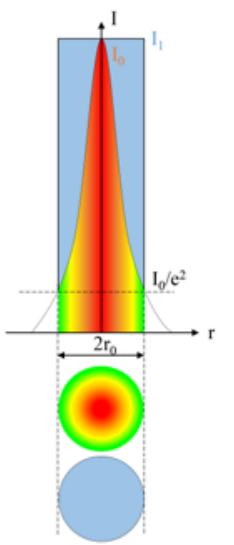

$\mathrm{I}_{0}$ Gaussian laser intensity

$I_{1}$ Top-hat laser intensity

\begin{tabular}{|c|c|}
\hline Parameter & Condition \\
\hline Glass transition temperature $T_{g}$ & $1475 \mathrm{~K}$ [18] \\
\hline Liquidus temperature $T_{l}$ & 1986 K $[18,19]$ \\
\hline Evaporation temperature $T_{e}$ & 2503 K [1] \\
\hline Density $\rho$ & $2201 \mathrm{~kg} / \mathrm{m}^{3}[1]$ \\
\hline Latent heat $\mathrm{Lm}_{\mathrm{m}}$ & 120 kJ/mol [1] \\
\hline Surface tension coefficient $\delta$ & 0.4 N/m [1] \\
\hline $\begin{array}{l}\text { Temperature derivative of surface } \\
\text { tension } \partial \gamma / \partial \mathrm{T}\end{array}$ & $-2.7 \cdot 10^{-4} \mathrm{~N} /(\mathrm{m} \bullet \mathrm{K})[1]$ \\
\hline Thermal expansion coefficient $\alpha$ & $5.5 \cdot 10^{-7} \mathrm{~K}^{-1}[1]$ \\
\hline Convective coefficient h & $10 \mathrm{~W} /\left(\mathrm{m}^{2} \bullet \mathrm{K}\right)[1]$ \\
\hline Surface emissivity $\varepsilon$ & $0.91[1]$ \\
\hline Initial temperature $T_{i}$ & $298.15 \mathrm{~K}[1]$ \\
\hline Ambient temperature $T_{a}$ & $289.15 \mathrm{~K}[1]$ \\
\hline
\end{tabular}

Spot radius

Fig.3 Intensity distribution of Gaussian and top-hat laser.

Table.1 Parameters and constants of the numerical model.

\section{Results}

\subsection{The temperature induced by Gaussian and top-hat} laser

The temperature on the surface with periodic topography of different periods ( $\mathrm{T}=5 \mu \mathrm{m}, 10 \mu \mathrm{m}, 25 \mu \mathrm{m}, 50 \mu \mathrm{m})$ and amplitudes $(\mathrm{A}=1 \mu \mathrm{m}, 5 \mu \mathrm{m}, 10 \mu \mathrm{m})$ were simulated. The temperature can be simulated within the softening and evaporation point by pre-setting irradiation time. The irra- 
diation time of Gaussian and top-hat was set $0-120 \mathrm{~ms}$ and 0-70 ms respectively. The surface temperature of molten pool was calculated.

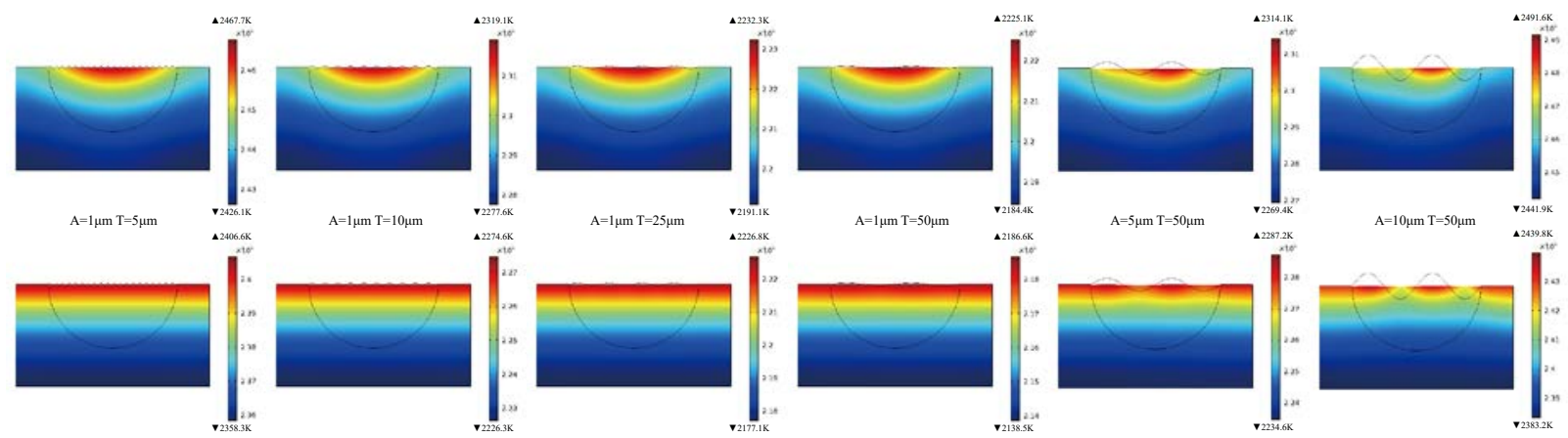

Fig.4 Surface temperature of molten pool subject to Gaussian and top-hat laser irradiation.

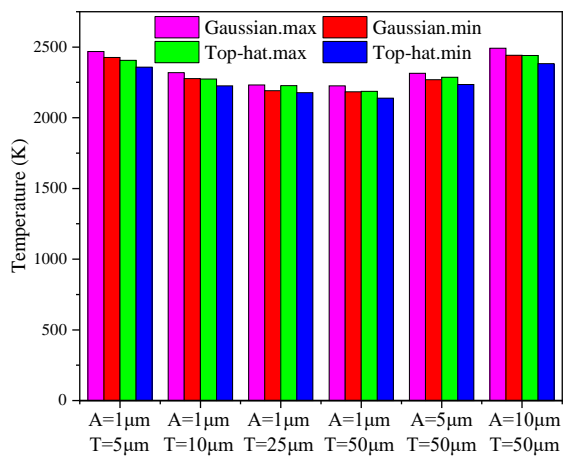

Fig.5 Surface temperature of molten pool.

Fig. 4 shows the temperature on surface of different parameters $(\mathrm{A}=1 \mu \mathrm{m} \mathrm{T}=5 \mu \mathrm{m}, \mathrm{A}=1 \mu \mathrm{m} \mathrm{T}=10 \mu \mathrm{m}, \mathrm{A}=1 \mu \mathrm{m}$ $\mathrm{T}=25 \mu \mathrm{m}, \mathrm{A}=1 \mu \mathrm{m} \mathrm{T}=50 \mu \mathrm{m}, \mathrm{A}=5 \mu \mathrm{m} \mathrm{T}=50 \mu \mathrm{m}, \mathrm{A}=10 \mu \mathrm{m}$ $\mathrm{T}=50 \mu \mathrm{m}$ ) under $120 \mathrm{~ms}$ Gaussian laser irradiation and 70ms top-hat laser irradiation. Fig.5 was drawn according to the maximum and minimum values of surface temperatures of molten pool under Gaussian and top-hat laser irradiation in Fig.4, making the extreme temperatures under different parameters more intuitive.

The temperature shows a downward trend with the increasing period. When the period T reaches $5 \mu \mathrm{m}$, the maximum temperature at the center region reaches $2467.7 \mathrm{~K}$ for Gaussian laser irradiation. The minimum temperature $2184.4 \mathrm{~K}$ appears at the period of $\mathrm{T}=50 \mu \mathrm{m}$. In addition, the surface temperature of different amplitudes ( $\mathrm{A}=1 \mu \mathrm{m}, 5 \mu \mathrm{m}$, $10 \mu \mathrm{m})$ at the same period ( $\mathrm{T}=50 \mu \mathrm{m})$ under $120 \mathrm{~ms}$ Gaussian laser irradiation was also shown in Fig.4. The results show that the temperature increases with the amplitude of periodic topography on initial surface. Obviously, the maximum temperature at the center region reaches $2225.1 \mathrm{~K}$ at the amplitude of $\mathrm{A}=1 \mu \mathrm{m}$. The maximum temperature is more likely to appear in the peak region of surface morphology. The surface morphology becomes more rough with the increase of amplitude A. It seems that the peak region will get laser radiation earlier than the valley region. Similar results can be observed in the simulation under tophat laser irradiation, as shown in Fig.4.

\subsection{Periodic topography evolution under Gaussian and top-hat laser}

To investigate how the laser intensity distribution (including Gaussian and top-hat laser) affects the surface morphology, the periodic topography evolution was simulated. The surface morphology and roughness (RMS) of periodic topography evolution with different periods ( $\mathrm{T}=5$ $\mu \mathrm{m}, 10 \mu \mathrm{m}, 25 \mu \mathrm{m}, 50 \mu \mathrm{m})$ and amplitudes $(\mathrm{A}=1 \mu \mathrm{m}, 5 \mu \mathrm{m}$, $10 \mu \mathrm{m})$ were modeled. The surface evolution of molten pool is shown in Fig. 7. It is cleat that the surface profile has altered upon Gaussian and top-hat laser irradiation. The surface roughness (RMS) of $100 \mu \mathrm{m}$ sampling was calculated using the formula:

$$
\mathrm{RMS}=\sqrt{\sum_{i=1}^{N} \frac{x_{i}^{2}}{N}}
$$

where $\mathrm{x}_{\mathrm{i}}$ represents the value of each point as well as $\mathrm{N}$ represents the number of points.
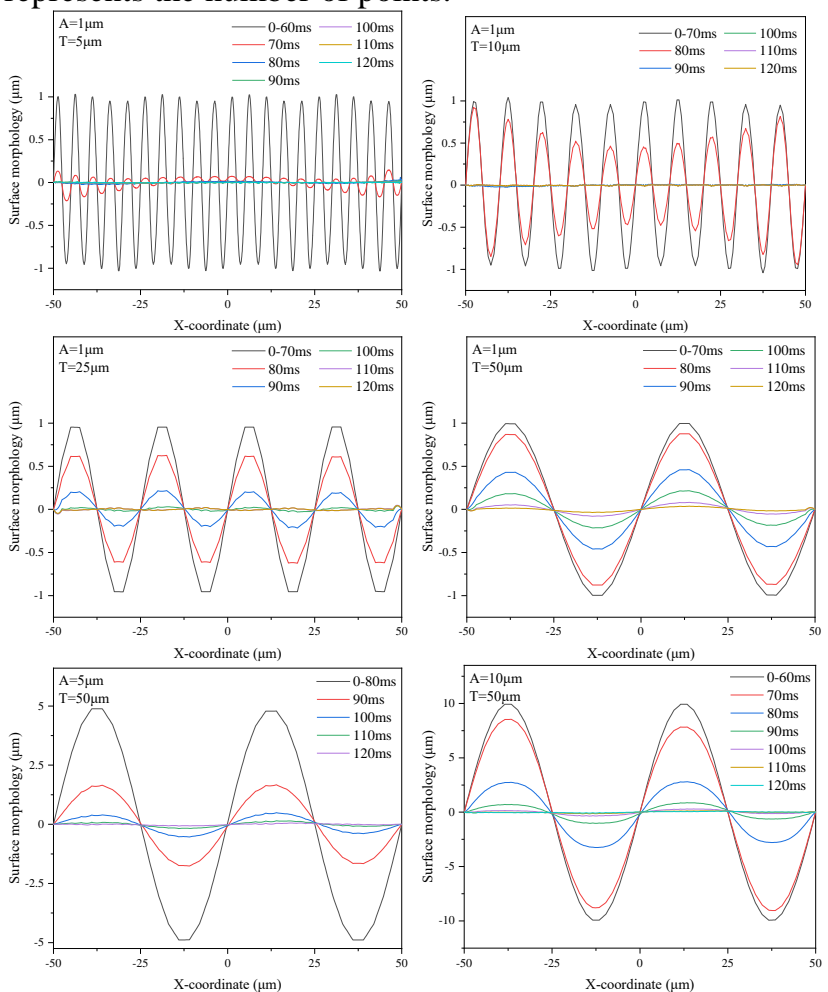

Fig.6 Surface evolution of molten pool under Gaussian laser.

When the temperature of fused silica reaches the liquefying temperature $(1986 \mathrm{~K})$, the phase transformation gradually takes place from solid to liquid, but the final temperature is lower than the evaporation temperature 
(2503 K). In the liquid state, the surface tension combining Marangoni effect will start to smooth the fused silica surface.
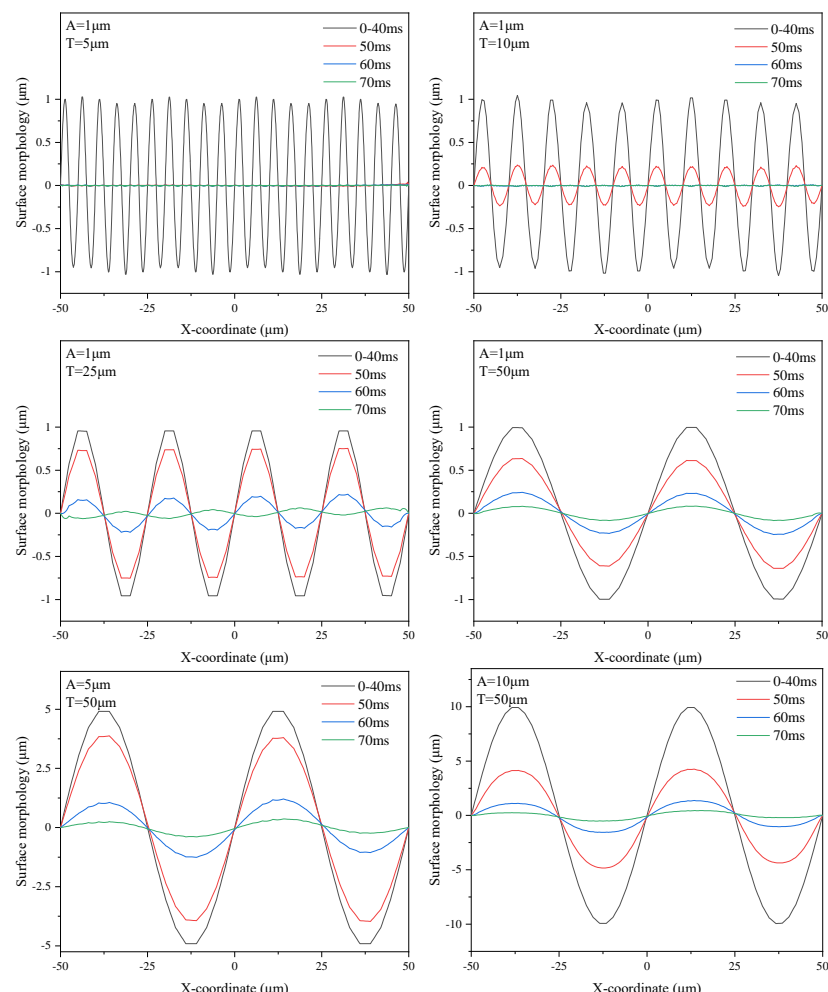

Fig.7 Surface evolution of molten pool under top-hat laser.

The simulation results showed that all the initial surfaces with different periodic topography can be smoothed. It is apparent from Fig.6, for Gaussian laser, the smooth velocity appears a downward trend with the increasement of period. Under the amplitude $\mathrm{A}=1 \mu \mathrm{m}$, the trigger time of the smoothing effect for period $\mathrm{T}=5 \mu \mathrm{m}$ was $70 \mathrm{~ms}$, and it was about $80 \mathrm{~ms}$ for $\mathrm{T}=10 \mu \mathrm{m}, 25 \mu \mathrm{m}, 50 \mu \mathrm{m}$. Although the $\mathrm{T}=50 \mu \mathrm{m}$ surface also starts to smooth at $80 \mathrm{~ms}$, its amplitude only decreases from $1 \mu \mathrm{m}$ to $0.8 \mu \mathrm{m}$, while for $\mathrm{T}=25$ $\mu \mathrm{m}$ to $0.6 \mu \mathrm{m}, \mathrm{T}=10 \mu \mathrm{m}$ can even to about $0.5 \mu \mathrm{m}$. In addition, when period $\mathrm{T}$ reaches $50 \mu \mathrm{m}$, the trigger time of smoothing effect for amplitude $A=1 \mu \mathrm{m}$ was $80 \mathrm{~ms}$, about 90 ms for amplitude $\mathrm{A}=5 \mu \mathrm{m}, 70 \mathrm{~ms}$ for amplitude $\mathrm{A}=10$ $\mu \mathrm{m}$. The trend of surface periodic morphology evolution is also applicable to top-hat laser from Fig.7, while the smooth velocity of top-hat laser is more rapidly, because top-hat laser can reach the smoothing temperature in a shorter time (compared with Gaussian laser). As for top-hat laser, the final smoothing time is $70 \mathrm{~ms}$, which is much less than that of Gaussian laser (120 ms). Under the amplitude $\mathrm{A}=1 \mu \mathrm{m}$, the initial smoothing time of period $\mathrm{T}=5 \mu \mathrm{m}$ is the fastest as $50 \mathrm{~ms}$. The amplitudes $\mathrm{A}=1,5$ and $10 \mu \mathrm{m}$ begin to smooth in $50 \mathrm{~ms}$ with period $\mathrm{T}=50 \mu \mathrm{m}$, but the smoothing range of the amplitude $\mathrm{A}=10 \mu \mathrm{m}$ is the largest, which decreases from $10 \mu \mathrm{m}$ to $4 \mu \mathrm{m}$.

The roughness (RMS) of periodic topography evolution during laser irradiation was also studied. Shown in Fig.8, 9 are the results for various surface morphology used in our simulation. As can be seen in Fig.8, 9, The change of roughness (RMS) is consistent with the trend of smoothing. No matter Gaussian or top-hat laser, the smoothing velocity of period $\mathrm{T}=5 \mu \mathrm{m}$ is more rapidly under the amplitude $\mathrm{A}=1$ $\mu \mathrm{m}$, correspondingly the roughness decreases faster. The largest reduction of roughness (RMS) occurred in period $\mathrm{T}=50 \mu \mathrm{m}$ and amplitude $\mathrm{A}=10 \mu \mathrm{m}$, where from initial $7060.9 \mathrm{~nm}$ decrease to $46.8 \mathrm{~nm}$ for Gaussian, to $290.7 \mathrm{~nm}$ for top-hat laser.

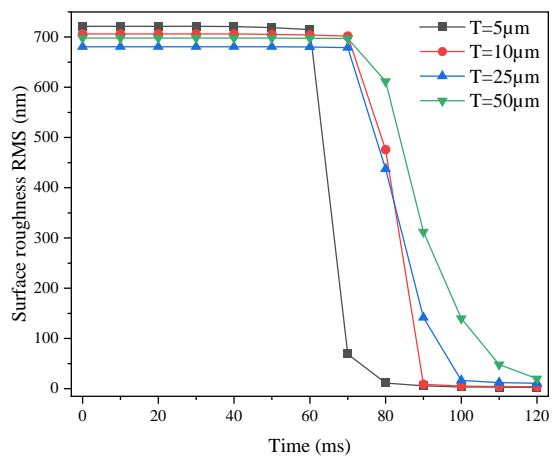

(a)

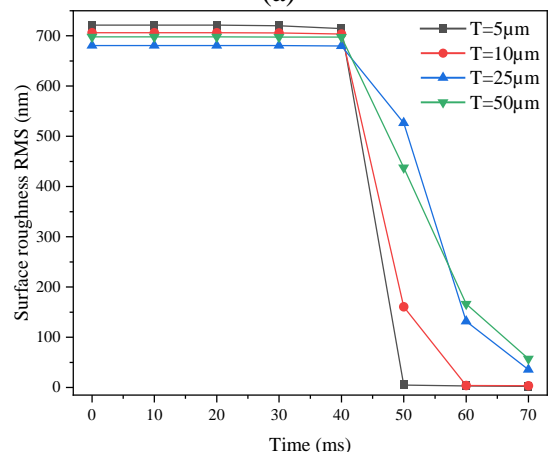

(b)

Fig.8 Roughness variation diagram when amplitude $\mathrm{A}=1 \mu \mathrm{m}$. (a) Gaussian;(b) Top-hat.

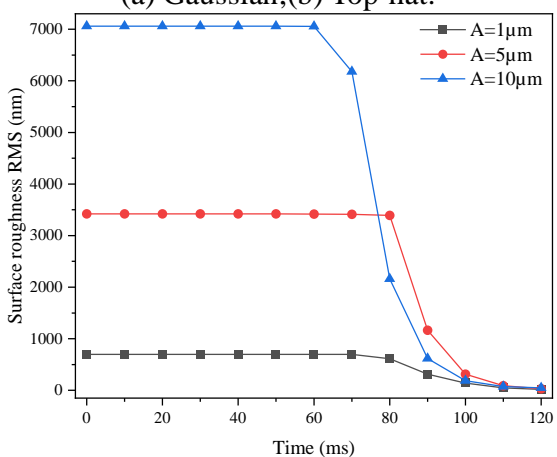

(a)

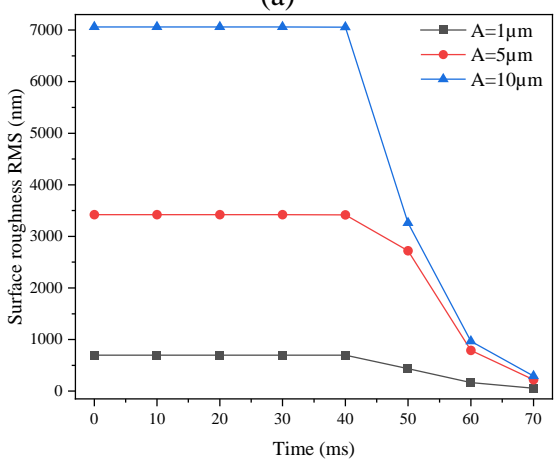

(b)

Fig.9 Roughness for different amplitude $(1 \mu \mathrm{m}, 5 \mu \mathrm{m}, 10 \mu \mathrm{m})$ with $\mathrm{T}=50 \mu \mathrm{m}$. (a) Gaussian;(b) Top-hat.

The roughness (RMS) reaches the lowest in $120 \mathrm{~ms}$ for Gaussian laser and $70 \mathrm{~ms}$ for top-hat laser. As shown in 
Fig.10, the best roughness (RMS) of Gaussian and top-hat is $2.85 \mathrm{~nm}$ and $2.48 \mathrm{~nm}$ respectively. In addition, when amplitude and period is altered, the optimal roughness (RMS) irradiated under Gaussian laser changes little with amplitude and period, but it showed significant difference as amplitude and period change for top-hat laser.

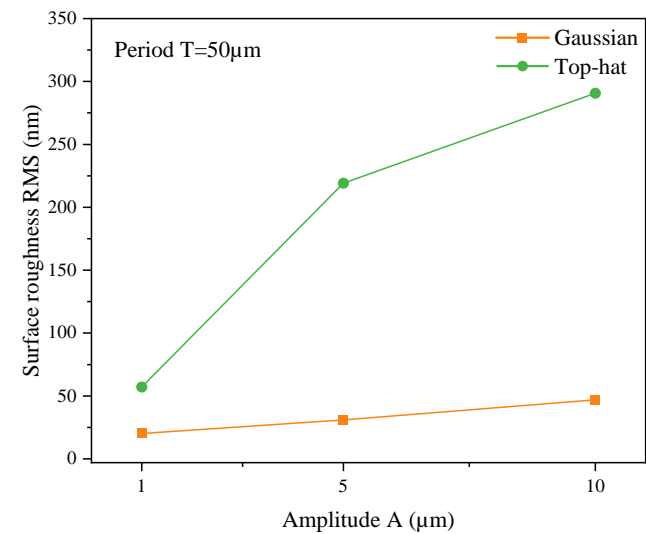

(a)

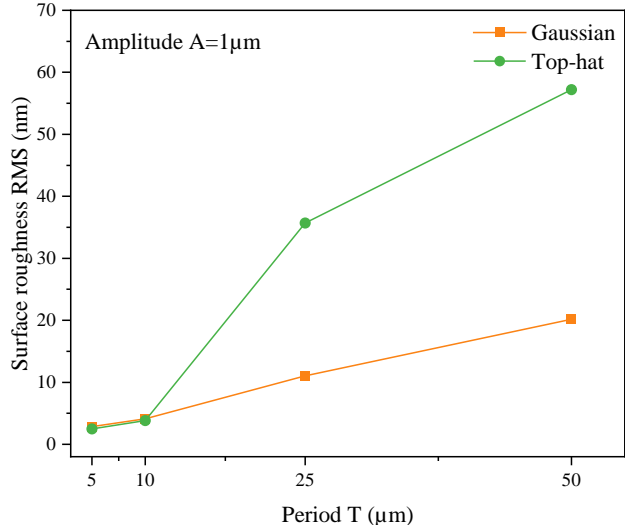

(b)

Fig.10 The surface roughness of molten pool when irradiated with Gaussian and top-hat laser. (a) Period $\mathrm{T}=50 \mu \mathrm{m}$ (b) Amplitude $\mathrm{A}=1 \mu \mathrm{m}$

\subsection{Random topography evolution under Gaussian and top-hat laser}

The random topography evolution was also simulated under Gaussian and top-hat irradiation. As shown in Fig.11, the initial uneven surface can be smoothed with the increasing laser irradiation time. Fig.12 shows the calculated surface roughness (RMS) after smoothing using Gaussian and top-hat laser. When laser irradiation time comes to an end, roughness (RMS) under Gaussian and top-hat changes very little, but for top-hat laser will cost less time to get optimal roughness (RMS) than that of Gaussian laser. The time to begin smoothing of top-hat laser is about $60 \mathrm{~ms}$, about 90 ms for Gaussian laser.

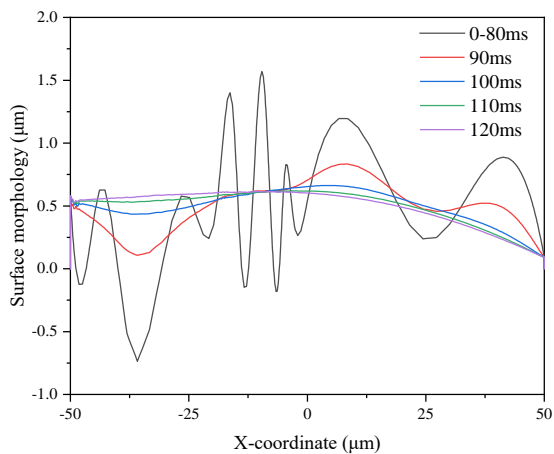

(a)

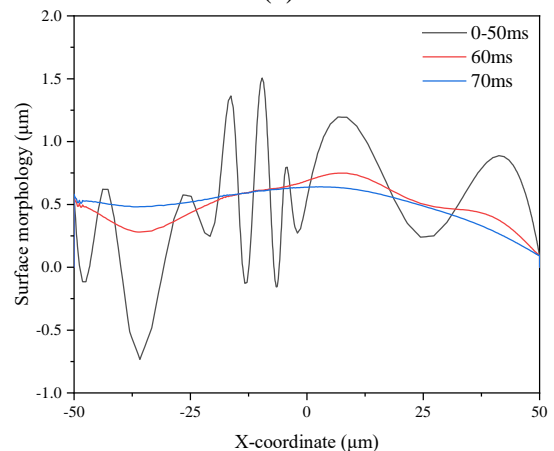

(b)

Fig.11 Random surface profile of molten pool. (a) Gaussian laser; (b) Top-hat laser.

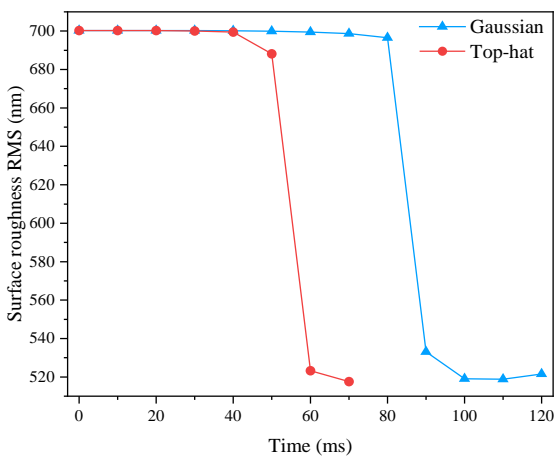

Fig.12 Surface roughness of molten pool.

\section{Discussion}

Fused silica strongly absorbs $10.6 \mu \mathrm{m}$ laser light within the topmost layers of tens of microns. About $80 \%$ of the absorbed laser can be converted into heat, resulting in sharp increase in local temperature. The temperature usually can reach as high as softening point or even evaporation temperature. It is necessary to control the laser parameters (mainly laser power, laser beam size and irradiation time) to ensure the temperature lie in between softening and evaporation temperature. For example, the temperature irradiated by Gaussian and top-hat laser was obtained in Fig.13 under the periodic molten pool profile with amplitude $\mathrm{A}=1 \mu \mathrm{m}$ and period $\mathrm{T}=5 \mu \mathrm{m}$. Furthermore, the irradiation time is mainly considered under the laser power density $585.69 \mathrm{~W} / \mathrm{cm}^{2}$. As shown in Fig.13-(a), when the Gaussian laser is applied for $84 \mathrm{~ms}$, the maximum temperature of molten pool surface appears at the center of molten pool, which is $1997 \mathrm{~K}$, and reaches the liquefying temperature (1986 K). With the passage of time, the area where the temperature rises above the liquidus temperature expands, and it completely enters the liquid state at $90 \mathrm{~ms}$. When the 
top-hat laser is applied from Fig.13-(b), the surface temperature of the molten pool is more uniform. The maximum temperature of molten pool surface is $1989 \mathrm{~K}$ with interaction time reaches $51 \mathrm{~ms}$, reaching the liquefying temperature (1986 K), and it completely enters the liquid state at 55 ms. It can be seen from the Fig.13 that the molten pool has transited from solid phase to liquid phase. Compared with the temperature of other models in Section3.1, all of them reach to liquefying temperature $(1986 \mathrm{~K})$ and lower than evaporation temperature (2503 K). Consequently, the stop time of simulation is $120 \mathrm{~ms}$ for Gaussian laser, $70 \mathrm{~ms}$ for top-hat laser.

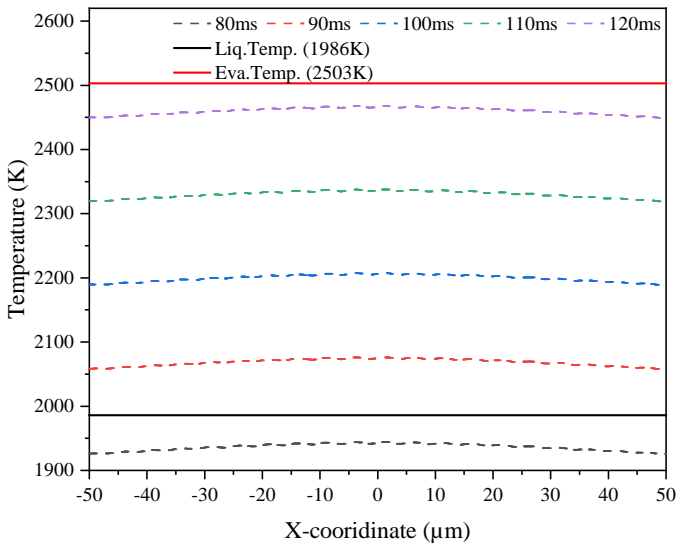

(a)

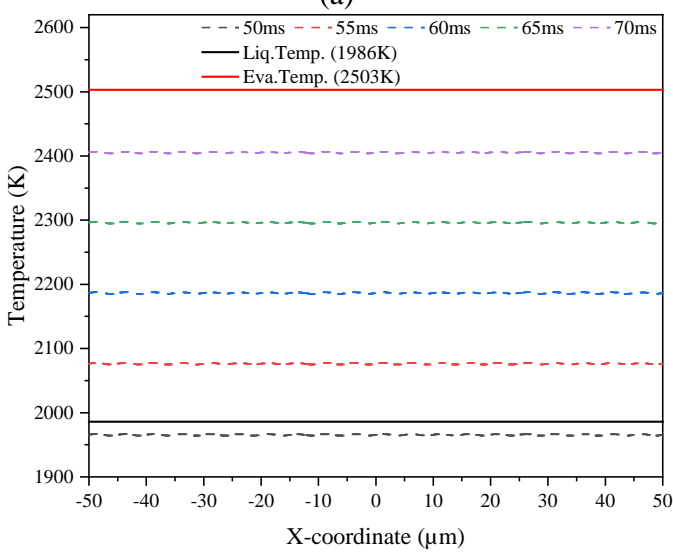

(b)

Fig.13 Surface temperature of molten pool. (a) Gaussian laser; (b) Top-hat laser.

Previous studies [15] have shown that there are two main driving forces for fluid flow: one is the surface tension related to the curvature of the surface profile, and the other is the Marangoni effect caused by the temperature gradient along the surface of the molten pool. If the tangential force caused by temperature gradient cannot overcome the viscous force, the surface tension (capillary force) controls the molten pool, which is called capillary zone; otherwise, Marangoni effect (thermocapillary force) dominates, which is called thermocapillary zone. These two effects are the main reasons for the smooth surface compared with other effects that like gravity etc. Consequently, the others are neglected.

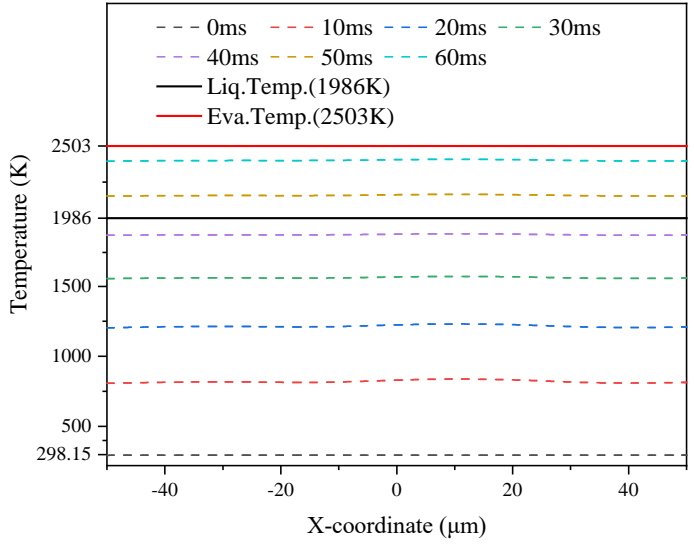

Fig.14 Surface temperature of validation model.

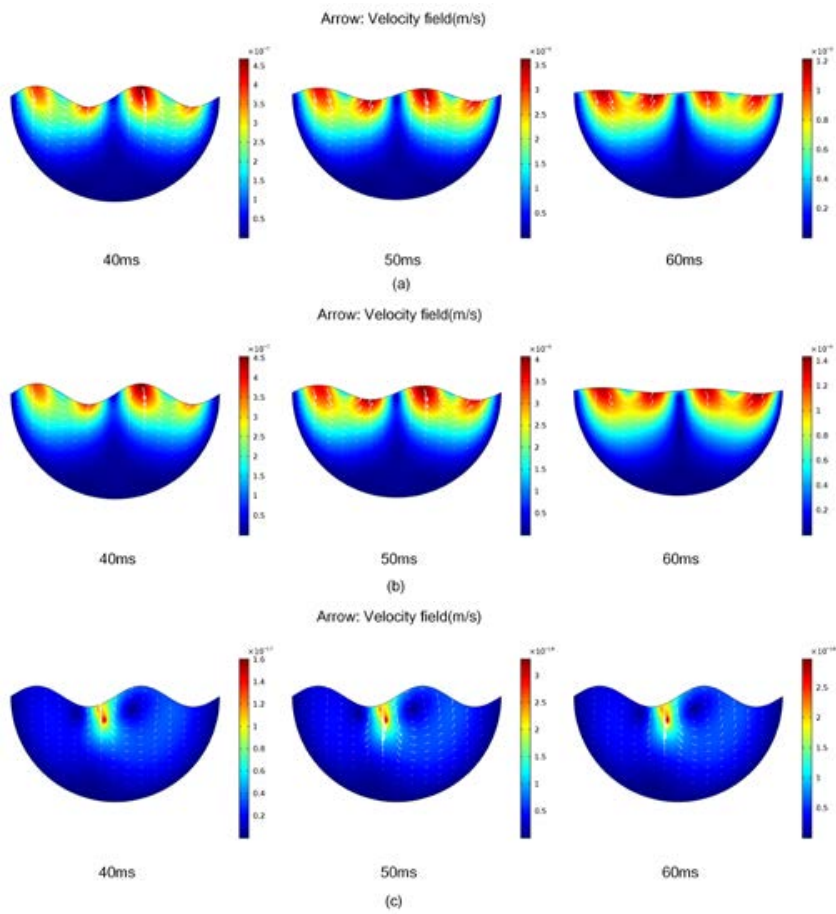

Fig.15 Velocity field and surface profile of validation model in 40-60 ms under Gaussian laser. (a) Synergic effect (Surface tension and Marangoni effect); (b) Surface tension (only); (c) Marangoni effect (only).

In order to simplify the calculation and intuitive expression, the periodic topography with amplitude $A=5 \mu \mathrm{m}$ and period $\mathrm{T}=50 \mu \mathrm{m}$ was selected, which was slightly different from the above model and called validation model for short The laser power and spot radius of validation model is 0.04 $\mathrm{W}$ and $50 \mu \mathrm{m}$ respectively. It can be seen from the Fig. 14 that the surface temperature of validation model has not reached the liquidus at $40 \mathrm{~ms}$, but it has been completely melted at $50 \mathrm{~ms}$ and $60 \mathrm{~ms}$. Therefore, we consider the surface morphology evolution of $\mathrm{V}$-model in 40-60 ms from Fig.15. The effects of surface tension and Marangoni effect on fused silica irradiated by Gaussian laser were simulated. The results show that when only the surface tension is applied, the molten material of convex surface (Peak) flows downward, and the molten material of concave surface (Valley) flows upward, and the large curvature surface morphology is eliminated. As shown in Fig.18(a) that the velocity field increases from $4.7 \times 10^{-7}$ $\mathrm{mm} / \mathrm{s}$ to $1.2 \times 10^{-4} \mathrm{~mm} / \mathrm{s}$ under the synergic effect (Surface 
tension and Marangoni effect), meanwhile the maximum velocity is $3.6 \times 10^{-4} \mathrm{~mm} / \mathrm{s}$ that appears at $50 \mathrm{~ms}$.

When the Marangoni effect is the only effect in the Fig.15-(c), the maximum velocity which appears at the center of the region reaches about $1.6 \times 10^{-17} \mathrm{~mm} / \mathrm{s}$ at $40 \mathrm{~ms}$, but the large curvature surface profile cannot be eliminated, and we found that the velocity field distribution from the edge to the center in the early formation of the molten pool, and from the center to the edge after the development of the molten pool, so that the molten material redistributes in the molten pool. It is the same as the conclusion of the previous simulation [13-15]. The surface morphology can be smooth under the surface tension is applied in the Fig.15 (b), but the maximum velocity will increase to $4.1 \times 10^{-4}$ $\mathrm{mm} / \mathrm{s}$. The function of surface tension is to make the molten material flows from the peak to valley. It is obvious that the maximum velocity appears at the peak of surface morphology, which is related to the higher temperature at the peak compared with valley region.

As shown in Fig.16, more detailed velocity field of validation model has been calculated under Gaussian laser irradiation. The velocity field tends to zero before the irradiation time of $44 \mathrm{~ms}$, and then suddenly increases to the maximum value of $5.2 \times 10^{-4} \mathrm{~mm} / \mathrm{s}$ with surface temperature above liquefying temperature $(1986 \mathrm{~K})$ at $46 \mathrm{~ms}$. The velocity will gradually decreases after $46 \mathrm{~ms}$. Similar tendency for the velocity field effected by surface tension can be observed obviously in Fig.16. The maximum value of velocity field increases to $5.4 \times 10^{-4} \mathrm{~mm} / \mathrm{s}$ at $47 \mathrm{~ms}$, but slower time that is $46 \mathrm{~ms}$ to achieve molten pool in the absence of Marangoni effect.

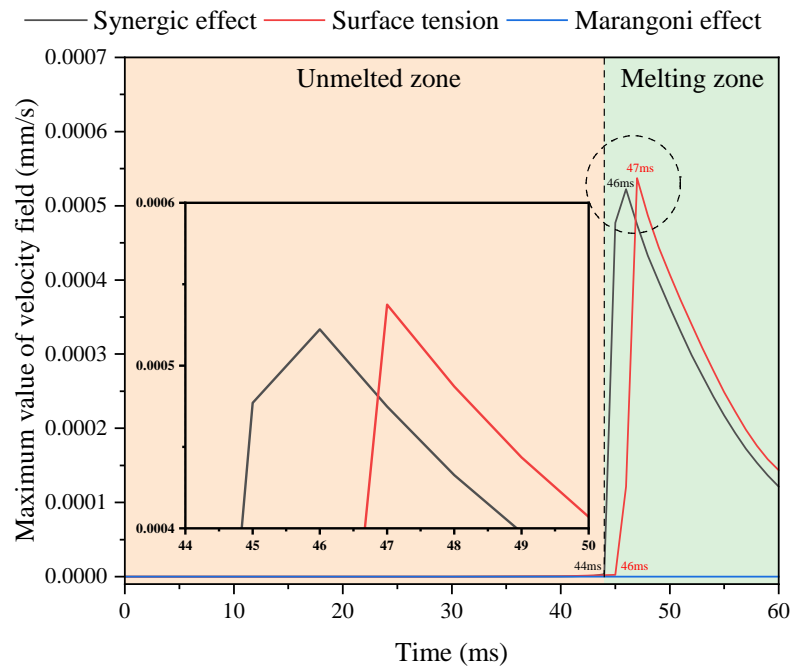

Fig.16 The maximum velocity field of validation model in 0-60 ms under Gaussian laser.

The validation model reveals the mechanism of laser polishing. First of all, the temperature of fused silica absorbing energy increases, leading to the transition from solid phase to liquid phase. In this process, it is necessary to control the temperature to make it below the evaporation temperature, otherwise it will be excessive to the gas phase. After the molten pool is completely melted, there will be surface tension and Marangoni effect on the surface. Under the combined action of these two forces, the large curvature surface morphology redistributes in the flow. The result is that the surface morphology becomes smooth, which is the same as the previous periodic surface morphology simulation results, and the random surface profile is also applicable.

\section{Conclusions}

In this paper, an improved simulation model of fused silica with $\mathrm{CO}_{2}$ laser was numerically simulated. The mechanism and internal process of laser polishing was analyzed by finite element simulation. The micro-flow mode of fused silica under different laser intensity distribution (Gaussian and top-hat) irradiation was investigated.

The temperature influenced by Gaussian and top-hat laser that appears a downward trend with the increase of period, increases with amplitude. Under the $585.69 \mathrm{~W} / \mathrm{cm}^{2}$ power density, the highest temperature of Gaussian and top-hat is $2467.7 \mathrm{~K}$ and $2406.6 \mathrm{~K}$ respectively at the amplitude $\mathrm{A}=1 \mu \mathrm{m}$ and period $\mathrm{T}=5 \mu \mathrm{m}$.

Top-hat laser is more efficient in smoothing the fused silica surface compared with Gaussian laser. Taking period $\mathrm{T}=50 \mu \mathrm{m}$ and amplitude $\mathrm{A}=1 \mu \mathrm{m}$ as example, the smoothing time for Gaussian laser is $80 \mathrm{~ms}, 50 \mathrm{~ms}$ for top-hat laser. Both Gaussian and top-hat laser can significantly reduce the surface roughness (RMS) of fused silica. The optimal roughness (RMS) of Gaussian and top-hat is $2.85 \mathrm{~nm}$ and $2.48 \mathrm{~nm}$ respectively in period $\mathrm{T}=5 \mu \mathrm{m}$ and amplitude $\mathrm{A}=1$ $\mu \mathrm{m}$.

The surface tension plays an important role in the surface smoothing process, and the Marangoni effect makes the molten material redistribute in the micro-flow. Under the combined action of these two forces, the super smooth surface can be achieved. The velocity field increases from $1.29 \times 10^{-13} \mathrm{~mm} / \mathrm{s}$ to $1.22 \times 10^{-4} \mathrm{~mm} / \mathrm{s}$ with temperature increases.

\section{Acknowledgments}

The authors acknowledge the funding of National Natural Science Foundation of China (No. 62175221), Consolidation Program for Fundamental Research (2019JCJQZDXX00), Foundation for Youth Talents of LFRC, CAEP (Grant No. LFRC-PD012), and Foundation for Scientific and Technological Innovations of CAEP (Grant No. CX2019025).

\section{References}

[1] D. Wang, F. Fan, M. Liu, T. Tan, H. Li, and Y. Li: Opt. Laser Technol., 127, (2020) 106141.

[2] Y.M. Xiao and M. Bass: Appl. Opt., 22, (1983) 2933.

[3] C. Buerhop, B. Blumenthal, R. Weissmann, N. Lutz, and S. Biermann: Appl. Surf. Sci., 46, (1990) 430.

[4] A. Richmann, E. Willenborg, and K. Wissenbach: Proc. OFT 2010, (2010) OTuC2.

[5] S. Heidrich, E. Willenborg, and A. Richmann: Phys. Procedia., 12, (2011) 519.

[6] S. Heidrich, A. Richmann, P. Schmitz, E. Willenborg, K. Wissenbach, P. Loosen, and R. Poprawe: Opt. Lasers Eng., 59, (2014) 34.

[7] C. Weingarten, A. Schmickler, E. Willenborg, K. Wissenbach, and R. Poprawe: J. Laser Appl., 29, (2017)

011702. 
[8] C. Weingarten, E. Uluz, A. Schmickler, K. Braun, E. Willenborg, A. Temmler, and S. Heidrich: Appl. Opt., 56, (2017) 777.

[9] S. Heidrich, A. Richmann, and E. Willenborg: Proc. SPIE, Vol. 8433 (2012) 84330P.

[10] Z. Yuan, Y. Li, D. Wang, T. Tan, H. Jin, Q. Zhang, J. Wang, and Q. Xu: Proc. SPIE, Vol. 11063, (2019) 1106316.

[11] C. Chan, J. Mazumder, and M.M. Chen: Metall. Mater. Trans. A, 15, (1984) 2175.

[12] R. Rai, J.W. Elmer, T.A. Palmer, and T. Debroy: J. Phys. D: Appl. Phys., 40 (2007) 5753.

[13] C. Ma, M. Vadali, N.A. Duffie, F.E. Pfefferkorn, and X. Li: Proc. MSEC 2013, (2013) 1117.

[14] C. Ma, M. Vadali, X. Li, N.A. Duffie, and F.E. Pfefferkorn: J. Micro Nano-Manuf., 2, (2014) 021010.

[15] C. Zhang, J. Zhou, and H. Shen: J. Manuf. Sci. Eng., 139, (2017) 041019.

[16] T. He, C. Wei, Z. Jiang, Y. Zhao, and J. Shao: Opt. Lett., 43, (2018) 5777.

[17] T. Doualle, L. Gallais, P. Cormont, D. Hébert, P. Combis, and J.-L. Rullier: J. Appl. Phys., 119, (2016) 113106.

[18] C.E. Protasov, R.S. Khmyrov, S.N. Grigoriev, and A.V. Gusarov: Int. J. Heat Mass Transf., 104, (2017) 665674.

[19] F. Nürnberg, B. Kühn, and K. Rollmann : Proc. SPIE, Vol. 10016, (2016) 100140F.

(Received: May 6, 2021, Accepted: September 18, 2021) 